\title{
EVALUATING THE EFFECTIVENESS OF PHONOPHORESIS BY PIROXICAM AND DIMETHYL SULFOXIDE FOR WOMEN'S WITH OSTEOARTHRITIS KNEE JOINT
}

\author{
MONISHA R ${ }^{1 *}$, MANIKUMAR $\mathbf{M}^{2}$, APARNA KRISHNAKUMAR ${ }^{3}$ \\ ${ }^{1}$ Department of Physiotherapy, SRM College of Physiotherapy, Kancheepuram, Tamil Nadu, India. ${ }^{2}$ Department of Physiotherapy, Saveetha \\ College of Physiotherapy, Chennai, Tamil Nadu, India. ${ }^{3}$ Department of Physiotherapy, Meenakshi College of Physiotherapy Chennai, Tamil \\ Nadu, India. Email: monishaphysio186@gmail.com \\ Received: 04 January 2018, Revised and Accepted: 17 March 2018
}

ABSTRACT

Objective: Osteoarthritis $(\mathrm{OA})$ is a progressive chronic disease with the loss of articular cartilage. In managing osteoarthritis, inadequate pain relief often occurs, particularly with a single non-steroidal anti-inflammatory drugs therapy.

Methods: A total of 50 participants were randomly allocated into three groups, received phonophoresis with piroxicam, dimethyl sulfoxide (DMSO), and ultrasound (US) therapy with aquasonic gel.

Results: On comparing, the baseline phonophoresis group with piroxicam showed significantly more pain reduction than the DMSO and US therapy. Enrolled patients in three groups have completed the study without any drawbacks.

Conclusion: This study showed that phonophoresis was superior to conventional US therapy in reducing pain in patients with symptomatic knee OA of a mild to moderate degree.

Keywords: Piroxicam, Dimethyl sulfoxide, Ultrasound therapy, Phonophoresis, Osteoarthritis.

(C) 2018 The Authors. Published by Innovare Academic Sciences Pvt Ltd. This is an open access article under the CC BY license (http://creativecommons. org/licenses/by/4. 0/) DOI: http://dx.doi.org/10.22159/ajpcr.2018.v11i6.24615

\section{INTRODUCTION}

Osteoarthritis (OA) is a progressive chronic disease with the loss of articular cartilage. In managing $\mathrm{OA}$, inadequate pain relief often occurs, particularly with single non-steroidal anti-inflammatory drugs (NSAIDs) therapy [1]. OA is the joint inflammation and degenerative process that have a direct influence on limiting the quality of life and well-being. Pain is the primary complaint experienced by patients, and it has the consequence of reducing the joint range of motion [1a]. Symptoms will be prevalent while engaging with weight-bearing activities such as walking, climbing stairs, and standing from a chair [2]. Sit to stand is the common activity of daily living performed by everyone, limitations in this may add burden that impacts people's quality of life and reliance on health-care needs [1].

Management of $\mathrm{OA}$ is reducing joint pain, as well as improving joint range and health-related quality of life. The traditional form of treatment for OA knee is the usage of wax therapy in addition to liquid paraffin, international recommendation on $\mathrm{OA}$ association has recommended a combination of pharmacologic treatment modalities in patients with knee OA [3]. European League against rheumatism suggested the usage of thermal modalities along with topical application of NSAIDs minimizes the adverse effects of oral ingestion of NSAIDs [4].

Dimethyl sulfoxide (DMSO) is odorless liquid produced as a byproduct of the paper industry. A limited number of small clinical trials indicate that dermal application of DMSO seems to provide rapid, temporary, and relief of pain in patients with arthritis [5]. There is no evidence that DMSO can alter progression of degenerative disease, and clinical trials are still lacking on the use of DMSO along with thermal modalities in the management of $\mathrm{OA}$ knee joint pain. Piroxicam is an NSAID used in musculoskeletal and joint disorders; piroxicam is more formulated in pharmaceutical industries because of their oil-free and water-removable desirability, efficacy, and harmless dermal effects [6].
The most adverse effects of piroxicam are reported at gastrointestinal track, and bleeding may occur with piroxicam suppositories [7]. Recent literature search has identified that application of a topical NSAID in combination with ultrasound (US) to improve the drugs absorption, a technique known as phonophoresis, may improve pain relief [4]. Phonophoresis is a form of physical therapy treatment. It commonly used for inflammatory conditions and other soft tissue injuries. Phonophoresis delivers drug from skin to subcutaneous tissues through US in combination with piroxicam gel and DMSO. It helps to reduce pain and improves joint range; the principal underneath the passage of drug by phonophoresis is by rapid movements [8].

The absorption of the drug and the delivery of the drug depend on vascular supply of the area [9]. Piroxicam and DMSO are applied to the US head onto the skin. Duration of the treatment which depends on the treatment area, if there is a large treatment area, it can be divided into equal half's, and the treatment has been commenced following that [10] local skin anesthesia, skin burns, mental illness, and skin irritation, in particular, treatment area are contraindicated for phonophoresis.

Knee OA is a common disabling chronic disease globally [11]. OA is a non-inflammatory joint disease, it affects all joints most commonly affected are hip and knee joints, and the affected age group is elderly people. OA is slowly progressive form of degenerative disease that causes cartilage loss morphophysiological damage of joints on early stage of $\mathrm{OA}$ and the more biochemical change in progress [12]. The most common signs and symptoms include pain, tenderness, joint stiffness, loss of flexibility, and bone spur, most commonly females are affected. Obesity age and joint injury and certain occupation, genetics, and bone deformity are the risk factor of OA [13].

Participants

All the participants filled their written informed consent. Participants registered in the study consisted of 65 women, in the age group of 
Table 1: Mean difference between phonophoresis and US therapy for VAS score

\begin{tabular}{lllll}
\hline Outcome measures & Pre-test & & & Post-test \\
\hline VAS & Group A & Group B & Group C & Group A \\
& 75 & 76 & 73 & 25 \\
p $\leq 0.00^{*}$ & & & Group B $\leq 0.00^{*}$ & 45 \\
\hline
\end{tabular}

VAS: Visual analog scale, US: Ultrasound

40-70 years, who fulfilled the American College of Rheumatology criteria for OA of the knee. As a baseline assessment, the visual analog scale (VAS) score was analyzed. During this assessment period, five patients were excluded because of their history of chronic inflammatory diseases, 10 women's were dropped out due to their allergic reaction to piroxicam.

\section{Study design}

Randomized controlled trial was conducted in a group of patients, and the therapist and patient were double blinded. After signing the consent, the participants who were met the inclusion criteria. A total of 50 participants were randomly allocated into three groups, Group A received phonophoresis with piroxicam, Group B is treated with DMSO, and Group C is treated with aquasonic gel as a coupling media and received US therapy. As the therapist and the patients were blinded, the coupling media, piroxicam, and DMSO were packed in the tubing, without any identification marks and the tubes were assigned numbers. The participants were not informed either they belong to Groups A, B, or C.

\section{Treatment}

Selection criteria

Inclusion criteria

- Women in the age group of 40-70 years.

- Baseline VAS score of 10.

Exclusion criteria

- Knee pain because of tendonitis, bursitis

- Chronic systemic inflammatory diseases

- History of allergy to piroxicam

- History of allergy to DMSO

- History of knee injury or surgery

- Visual analog score $<10$

- Patients who are medicated with NSAIDs, corticosteroids.

\section{Group: A - Phonophoresis with piroxicam}

The coupling media is prepared by mixing the piroxicam gel with a standard coupling agent at a ratio of 4:10. It is applicable to fulfill the blinding process as the gel is same in color and lack odor. During the treatment, patients were not told about the components of the coupling media. Before initiating the treatment, skin is rubbed with alcohol.

\section{Treatment parameters}

- Mode: The continuous mode

- Power: $1 \mathrm{w} / \mathrm{cm}^{2}$

- Frequency: $1 \mathrm{MHZ}$

- Method: Stroking

- Treatment duration: 10 min.

\section{Group: B - Phonophoresis with DMSO}

The coupling media is prepared by mixing the DMSO gel with a standard coupling agent at a ratio of $4: 10$. It is applicable to fulfill the blinding process as the gel is same in color and lack odor. During the treatment, patients were not told about the components of the coupling media. Before initiating the treatment, skin is rubbed with alcohol.

\section{Treatment parameters}

- Mode: The continuous mode

- Power: $1 \mathrm{w} / \mathrm{cm}^{2}$
- Frequency: $1 \mathrm{MHZ}$

- Method: Stroking

- Treatment duration: $10 \mathrm{~min}$ and total sessions: 10 (5 times a week).

Group: C - US therapy with aquasonic gel

The coupling media is a simple aquasonic gel. The purpose of the Group C was to analyze the effect of US alone without the addition of piroxicam and DMSO.

\section{Treatment parameters}

- Mode: The continuous mode

- Power: $1 \mathrm{w} / \mathrm{cm}^{2}$

- Frequency: $1 \mathrm{MHZ}$

- Method: Stroking

- Treatment duration: $10 \mathrm{~min}$.

Outcome measures

Every patient was assessed at the baseline including age, weight, height, duration of knee pain, and the side of the knee that was more painful. The outcomes were measured at the baseline and 2 days after the last treatment session. To avoid the immediate and short-term effects of heat application, post-treatment measures were performed $2 \mathrm{~h}$ later after the program finished. The outcome measured was reduction of pain which is assessed using a VAS.

\section{Statistical analysis}

Analyses were performed using SPSS statistical software, baseline characteristics between the phonophoresis and US groups were compared using the independent $\mathrm{t}$-test. The Wilcoxon signed-rank test was used to assess within-group changes for VAS. On comparing, the baseline phonophoresis group with piroxicam showed significantly more pain reduction than the DMSO and US therapy. Enrolled patients in three groups have completed the study without any drawbacks.

\section{DISCUSSION}

Patients with OA knee may experience adverse effects because of oral NSAIDs. There is a long-standing interest in the development of some non-pharmacological management for pain reduction in OA knee population.

Topical application of NSAIDs is effective for pain reduction, if the delivery of drug is enhanced by some electrotherapeutic means. US is the acoustic vibration propagated in the form of sonic waves, application of electrotherapeutic agent helps to eliminate the barrier (skin) for NSAIDs transmission for pain relief. US therapy coupled with piroxicam is referred as phonophoresis, is used as an enhancer for drug delivery. However, physiotherapy clinics use phonophoresis, but the evidence on the treatment methodology is still scarce. However, improvement in knee function with phonophoresis is greater than with US. From this analysis, it would be shown that phonophoresis is the appropriate treatment for patients with $\mathrm{OA}$ with piroxicam as the coupling media [Table 1]. VAS score shows a significant change from the baseline in Group A (70\%) on comparing Group B (65\%) and C (50\%). Pain has been reduced by about $70 \%$ in 2 weeks, and it is also noted that pain relieved with phonophoresis is twice as greater than US therapy. Patients are advised with simple quadriceps strengthening exercises as a home exercise program. The results from this study differed from those of a previous study [4] which concluded that ibuprofen phonophoresis was not superior to conventional US in patients with knee OA. The treatment procedures that operated in the study induced 
questionable concepts such as the preparation of ibuprofen cream, duration of treatment per session, and recruitment of participants with a severe grading of OA (Kellgren-Lawrence Grade IV), which inspired to generate the hypothesis of this study [14-18]. Identifying the superiority of the phonophoresis technique to conventional US in $\mathrm{OA}$ was conducted using the piroxicam gel, $10 \mathrm{~min}$ of treatment per session and mild to moderate degree of knee OA. Results from this study showed that phonophoresis was superior to conventional US and also confirmed the findings of previous reports regarding factors influencing the quality and efficacy of phonophoresis $[11,19]$.

Future studies are required to clarify the parameters of US for effectively facilitate topical drug diffusion, what US duration should be used to maximize absorption of the drug, and which topical drugs can be most effectively used for phonophoresis.

\section{CONCLUSION}

This study showed that phonophoresis was superior to conventional US therapy in reducing pain in patients with symptomatic knee OA of a mild to moderate degree.

\section{AUTHOR'S CONTRIBUTION}

M. Manikumar participated in the design of the study, Aparna Krishnakumar K performed the statistical analysis and contributed to draft the manuscript, participated in the design of the study and the gathering of data, and Monisha. $\mathrm{R}$ contributed to the design of the study, collaborated in gathering of data, and helped to draft the final manuscript.

\section{CONFLICT OF INTEREST}

Authors declare they have no conflict of interest and no funding has been obtained from any source.

\section{REFERENCES}

1. Oka Dwicandra NM, Setiadi AA, Comparison of pain score in osteoarthritis patients treated with a combination of diacerein and meloxicam and meloxicam alone. Int J Pharm Pharm Sci 2017;9:69-73. (a) Kotlarz H, Gunnarsson CL, Fang H, Rizzo JA. Insurer and out-of pocket costs of osteoarthritis in the US: Evidence from national survey data. Arthritis Rheum 2009;60:3546-53.

2. Dunlop DD, Manheim LM, Song J, Chang RW. Arthritis prevalence and activity limitations in older adults. Arthritis Rheum 2001;44:212-21.

3. Zhang W, Moskowitz RW, Nuki G, Abramson S, Altman RD, Arden N, et al. OARSI recommendations for the management of hip and knee osteoarthritis, part II: OARSI evidence-based, expert consensus guidelines. Osteoarthritis Cartilage 2008;16:137-62

4. Rutjes AW, Nuesch E, Sterchi R, Juni P. Therapeutic ultrasound for osteoarthritis of the knee or hip. Cochrane Database Syst Rev2010;1:CD003132.

5. Zhang Y, Jordan JM. Epidemiology of osteoarthritis. Clin Geriatr Med 2010;26:355-69.

6. Soni A, Kiran A, Hart DJ, Leyland KM, Goulston L, Cooper C, et al. Prevalence of reported knee pain over twelve years in a communitybased cohort. Arthritis Rheum 2012;64:1145-52.

7. Zhang W, Doherty M. EULAR recommendations for knee and hip osteoarthritis: A critique of the methodology. Br J Sports Med 2006;40:664-9.

8. Tascioglu F, Kuzgun S, Armagan O, Ogutler G. Short-term effectiveness of ultrasound therapy in knee osteoarthritis. J Int Med Res 2010;38:1233-42.

9. Paliwal S, Mitragotri S. Therapeutic opportunities in biological responses of ultrasound. Ultrasonics 2008;48:271-8.

10. Chung JI, Barua S, Choi BH, Min BH, Han HC, Baik EJ, et al. Antiinflammatory effect of low intensity ultrasound (LIUS) on complete freund's adjuvant-induced arthritis synovium. Osteoarthritis Cartilage 2012;20:314-22.

11. Ateia YA, Al-Edanni MS, Al-Qurtas MS. Impact of metformin and serratiopeptidase in obese patients with knee osteoarthritis. Int J Pharm Pharm Sci 2018; 10:37-41.

12. Deshpande MM, Patil CB. Heel pain and phonophoresis. J Indian Med Assoc 2010;108:365.

13. Yildiz N, Atalay NS, Gungen GO, Sanal E, Akkaya N, Topuz O, et al. Comparison of ultrasound and ketoprofen phonophoresis in the treatment of carpal tunnel syndrome. J Back Musculoskelet Rehabil 2011;24:39-47.

14. Ay S, Doğan SK, Evcik D, Bașer OC. Comparison the efficacy of phonophoresis and ultrasound therapy in myofascial pain syndrome. Rheumatol Int 2011;31:1203-8.

15. Nagrale AV, Herd CR, Ganvir S, Ramteke G. Cyriax physiotherapy versus phonophoresis with supervised exercise in subjects with lateral epicondylalgia: A randomized clinical trial. J Man Manip Ther 2009; $17: 171-8$

16. Silveira PC, Victor EG, Schefer D, et al. Effects of therapeutic pulsed ultrasound and dimethylsulfoxide (DMSO) phonophoresis on parameters of oxidative stress in traumatized muscle. Ultrasound Med Biol 2010;36:44-50.

17. Vlak T. Comparative study of the efficacy of ultrasound and sonophoresis in the treatment of painful shouder syndrome. Reumatizam 1999:46:5-11.

18. Kozanoglu E, Basaran S, Guzel R, Guler-Uysal F. Short term efficacy of ibuprofen phonophoresis versus continuous ultrasound therapy in knee osteoarthritis. Swiss Med Wkly 2003;133:333-8.

19. Goraj-Szczypiorowska B, Zajac L, Skalska-Izdebska R. Evaluation of factors influencing the quality and efficacy of ultrasound and phonophoresis treatment. Ortop Traumatol Rehabil 2007;9:449-58. 